\title{
Symplectic Map Tracking for the LHC
}

\author{
D. T. Abell, BNL, \\ F. McIntosh and F. Schmidt, CERN
}

\begin{abstract}
Tracking simulation is the essential tool to evaluate how multipolar imperfections of the magnets limit the regime of stable particle motion in phase space. In the LHC, for instance, particles have to remain at injection energy, where the multipolar errors are at their maximum, for more than 10 million turns. Systematic tracking studies have to be limited to a small fraction of this total time even on modern computer systems. A considerable speed-up is expected when a symplectified one-turn map can be used instead of the element-by-element tracking. In this report we have applied this method for various map orders to the realistic case of the LHC lattice version 6 with special emphasis on precision and gain in speed.
\end{abstract}

\section{INTRODUCTION}

Since 1982 when maps have been introduced to the accelerator field [1] the question has been raised if one can use them to replace element-by-element tracking. It became clear that in the presence of strong multipolar fields maps can be used only if one applies some kind of symplectification scheme [2]. Such a scheme has been proposed in 1989 by J. Irwin [3]. For the case of the LHC this scheme has been tested [4] but found firstly, to be only a factor of 2 faster, and secondly, to be insufficiently precise with respect to the determination of the dynamic aperture. The application of this technique was therefore discontinued until recently when a new scheme has been proposed [5] that guarantees a minimal set of higher orders to achieve symplecticity. In this article we use a realistic model of the LHC to determine if this new approach allows a sufficiently precise determination of the dynamic aperture but with a tenfold speed-up compared to direct tracking. To this end we are studying 60 different random distributions of the multipolar components of main magnets (later called "seeds"). The involved theoretical background of this technique is described in an upcoming article soon to be published [6].

\section{CODE OPTIMISATION}

The implementation of Cremona map tracking requires three principal tools: (1) a means for constructing the Taylor map for a given machine lattice; (2) a routine for converting that Taylor map into a corresponding Cremona map; and (3) a routine for tracking with the resulting Cremona map. Tool 1 was already available in SIXTRACK's suite of tools [7]. Tool 2 was implemented in the program
CREMONA, which makes extensive use of Étienne Forest's LiELIB package [8]. Tool 3 was implemented in the program CTRACK. Because tracking consumes so much more time than map production, only the last tool, CTRACK, was subjected to intensive optimisation, details of which will be presented in Ref. [6].

Fig. 1 shows execution times (hollow symbols, left-hand ordinate) and speed gains (solid symbols, right-hand ordinate) versus map order for different aspects of Cremona map tracking as compared to direct tracking. Execution times are shown for creating a Taylor map (DA map), converting it to a Cremona map (C map), doing the actual tracking (C track), and the sum of these times (Total). The gains shown are just the ratios of the times required for direct tracking (shown by the horizontal line at the top) and Cremona map tracking-either "C track" (gain), or "Total" (net gain). It has to be mentioned that the execution time for the direct tracking consists of a "typical" LHC dynamic aperture run over $10^{5}$ turns, 2 amplitude ranges and 5 phase space angle variations (see Ref. [9]).

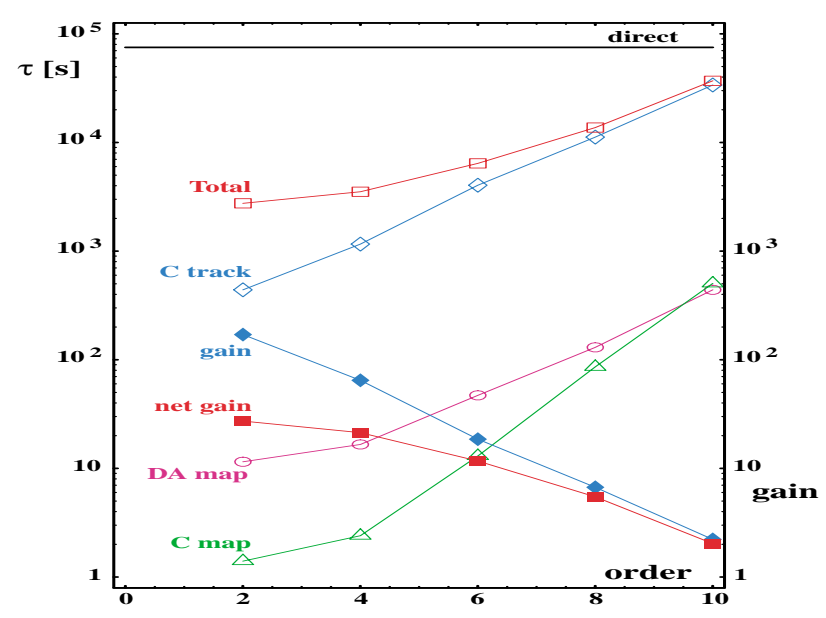

Figure 1: Execution times and speed gains versus map order for different aspects of Cremona map tracking.

The relevant quantity to evaluate Cremona map tracking against direct tracking is of course the achieved net gain. We can therefore conclude that the targeted speed-up of a factor of 10 can be reached for the map orders 6 to 8 where almost all time is spent in the final Ctrack part. At map order 10 the speed-up factor reduces to a factor of 2 which hardly justifies the effort of going through several preparatory steps. 

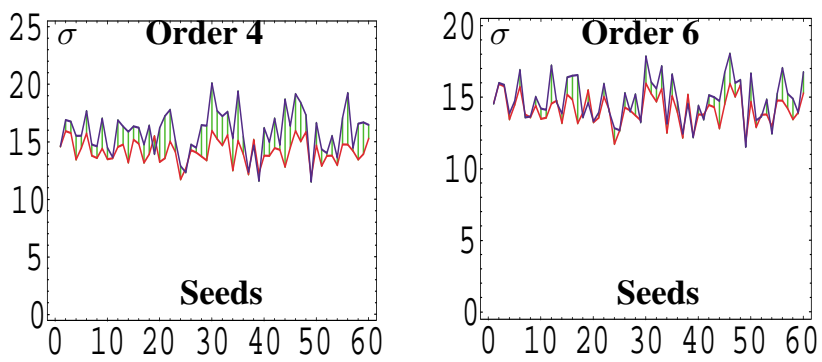
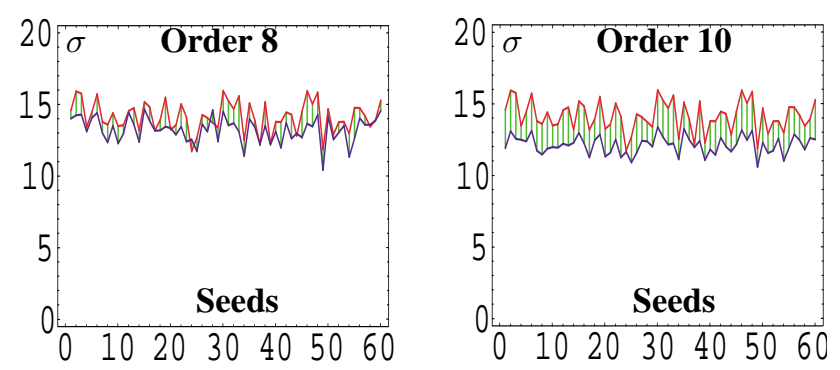

Figure 2: Dynamic aperture comparisons.

\section{CREMONA MAP PRODUCTION}

The LHC optics version 6, comprises a design (ideal) lattice together with sixty different seeds representing the range of imperfections expected to exist in the real machine. To build Cremona maps for each of these rings, we first used the available SIXTRACK tools [7] to construct tenth-order Taylor maps representing one turn around each of these sixty rings. Then the code CREMONA was used to construct the corresponding Cremona maps of (Taylor) orders 2, 4, 6, 8, and 10. In the six-dimensional case (with the RF cavity on) the constructed map carried particles around the ring from just after the RF cavity to just before it. The RF cavity was then treated as an exact kick. The virtue of this approach was that the "one-turn" map did not change the momentum deviation $\delta=\left(p-p^{0}\right) / p^{0}$, and we could therefore use a technique to greatly simplify the Cremona symplectification.

\section{RESULTS AND ANALYSIS FOR THE LHC OPTICS VERSION 6}

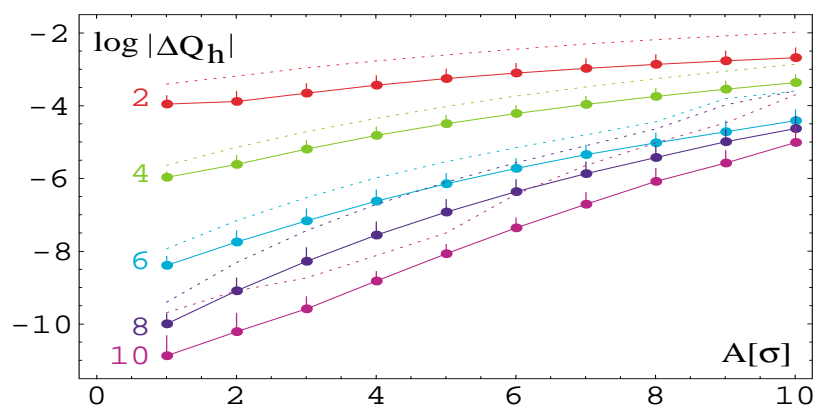

Figure 3: Horizontal tune error versus amplitude for different Cremona map orders.

To assess the utility of Cremona maps for long-term tracking studies of the LHC one might compare phasespace portraits generated by direct (element-by-element) and Cremona map tracking. This qualitative comparison would only demonstrate catastrophically large differences. The absence of these kind of differences is not sufficient to guarantee that more subtle effects, which may cause particle losses after long iteration periods, are well reproduced. For a more quantitative comparison between Cremona map tracking and direct tracking, we looked at the following measures: (1) differences in the amplitude-dependent tune, or detuning error; (2) differences in the predicted dynamic aperture (DA); (3) one-turn tracking errors. We describe each of these in turn.

From particle tracking data one can determine the tunes of a given particle. Fig. 3 shows the results of just such an analysis on particles launched at five different angles (in $x-y$ space) and at ten different amplitudes (from 1 to $10 \sigma$ ), and followed using direct tracking around one particular LHC lattice (seed 39). Using symbols, we say, for example, that each point of the left-hand plot represents a value for the tune $Q_{h}(A, \theta, r, \mathcal{X})$ of a particle launched with amplitude $A$ and angle $\theta$, and tracked around the LHC lattice, seed $r$, using direct tracking, indicated here by the "exact" map $\mathcal{X}$.

Fig. 3 shows how the results based on Cremona map tracking differ from those based on direct tracking. In, say, the left-hand plot of that figure, each point represents an average - over the sixty different seeds $r$ and the five different angles $\theta$-of the absolute error

$$
\left|Q_{h}\left(A, \theta, r, \mathcal{C}_{n}\right)-Q_{h}(A, \theta, r, \mathcal{X})\right|,
$$

where $\mathcal{C}_{n}$ denotes the Cremona map of (Taylor) order $n$. The error bars on the points indicate one standard deviation above the mean values, and the dashed lines indicate the maximum values. At small amplitudes the errors significantly decrease when the order of the map is increased. However, at large amplitudes of some $10 \sigma$ the errors are much larger but also the differences between the orders are much reduced. In fact, one can argue that at these large amplitudes it does not seem to be worthwhile to increase to more than the map order 6.

The second and most essential criterion is testing the quality of the prediction of the dynamic aperture, which is the main goal of the simulation studies. To compute the DA for a given lattice we simply search for the smallest amplitude at which the particle reaches very large amplitudes, i.e. the so called overflow criterion. Fig. 2 shows the dynamic aperture calculated for the the 60 seeds for the Taylor map orders 4, 6, 8 and 10. In red (the same in all 4 graphs) one finds the results from direct tracking while the Cremona map tracking is shown in blue; in green the differences are high-lighted. Note that for order 4 we are using a slightly different scale. As expected, the precision of the DA prediction improves from order 4 to 6 and for the latter the results are very satisfactory. However, unexpectedly the results become worse when the higher orders $8-10$ are used. 

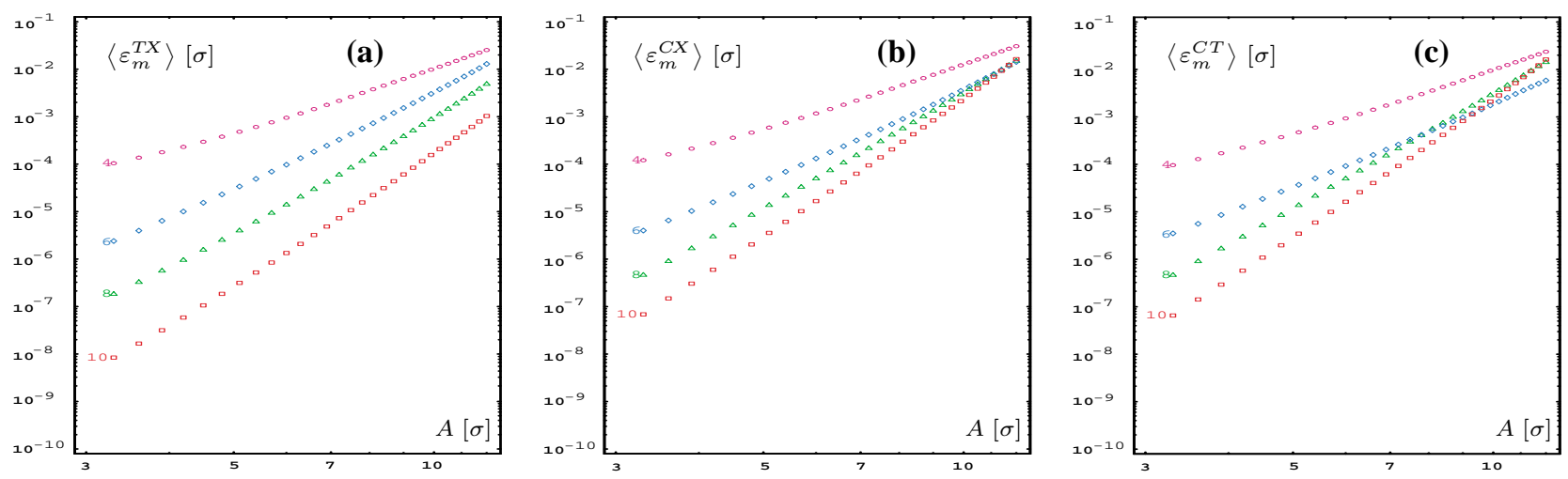

Figure 4: Average one-turn error $\left\langle\varepsilon_{m}^{\alpha}\right\rangle$ versus amplitude.

In the rest of this article we give an analysis of why higher orders seem not to be beneficial. The two measures described so far-detuning errors and differences in predicted DA-might be called "high-level" measures; they report how well Cremona map tracking performs the tasks we want it to do. By contrast, the next measure might be described as "low-level"; it reports how well Cremona map tracking performs on a turn-by-turn basis.

Recall, for a moment, the origin of our Cremona maps; from a tracking code, corresponding to some "exact" map $\mathcal{X}$, we extract a truncated Taylor series map $\mathcal{T}_{m}$ containing terms through order $m$, so that $\mathcal{T}_{m}=\mathcal{X}+O\left(z^{m+1}\right)$. This Taylor map is then symplectified by converting it to a Cremona map $\mathcal{C}_{m}$ that agrees with the Taylor map through the same order $m$; hence $\mathcal{C}_{m}=\mathcal{T}_{m}+O\left(z^{m+1}\right)$. It follows that the three maps $\mathcal{X}, \mathcal{T}_{m}$, and $\mathcal{C}_{m}$ all differ from one another by terms that scale as $z^{m+1}$; but we would like to know just how big those differences are. To that end we define the one-turn errors

$$
\begin{aligned}
& \varepsilon_{m}^{T X}(z)=\left\|\mathcal{T}_{m} z-\mathcal{X} z\right\|, \\
& \varepsilon_{m}^{C X}(z)=\left\|\mathcal{C}_{m} z-\mathcal{X} z\right\|, \\
& \varepsilon_{m}^{C T}(z)=\left\|\mathcal{C}_{m} z-\mathcal{T}_{m} z\right\|,
\end{aligned}
$$

where $\|\cdot\|$ denotes an appropriate vector norm on phase space. To determine in some meaningful way how the average errors vary with amplitude, we require at each amplitude a set of points over which to average. To generate a given set of points, we launch a single particle at the given amplitude (between 3 and $12 \sigma$ ) and use direct tracking to follow it for 1000 turns, thus generating the phase-space points

$$
\left\{z^{0}, z^{1}, z^{2}, \ldots, z^{1000}\right\}=\left\{z^{0}, \mathcal{X} z^{0}, \mathcal{X} z^{1}, \ldots, \mathcal{X} z^{999}\right\} .
$$

The first one-thousand of these, 0-999, constitute the desired set of points. Applying also the maps $\mathcal{T}_{m}$ and $\mathcal{C}_{m}$ to these points, we can compute the one-turn errors in (1), and average over the 1000 points. For the vector norm in (1) we used the Pythagorean length in horizontal phase space, and we show the results for relative momentum deviations $\delta=7.5 \times 10^{-4}$ in Fig. 4. As expected we find that the error between direct and the Taylor map tracking (part a) decreases with the order of the map and there is an ampli- tude dependent increase of the error approximately according to the proper power law. However, when we consider the error between the Cremona tracking with respect to direct and Taylor map tracking (part b and c respectively) we find that the error at small amplitude is not decreasing as rapidly with order as in part (a). This means that the symplectification method of adding higher order terms is less efficient in relative terms at higher orders. Since the errors have to increase with amplitude according to the same power laws it is clear that at some intermediate amplitude the higher orders lead to less precise results than the lower orders.

\section{SUMMARY}

We find that at order 6 (multipolar components are considered up to order 7) the targeted net speed gain of 10 over direct tracking can be achieved for the LHC. On the other hand, we have also shown that at order 6 the errors are at their minimum over the amplitudes of interest. We conclude that for the LHC where multipole components beyond order 7 are less relevant Cremona map tracking is an attractive mean for rapid systematic investigations.

Our advice for the potential client at other future accelerator is to simply examine the one-turn errors $\varepsilon_{m}^{\alpha}$, which can be done rapidly. From plots such as those in Fig. 4 one can determine whether or not Cremona map tracking will be useful.

\section{ACKNOWLEDGEMENTS}

One of us (DA) would like to thank the AP group of the CERN SL division for hospitality and support.

\section{REFERENCES}

[1] A. J. Dragt. AIP Conf. Proc. No. 87, 1982.

[2] R. Kleiss et al., Tech. Rep. CERN SL/92-02(AP).

[3] J. Irwin. AIP Conf. Proc. No. 326, 1995, SSC-228, 1989.

[4] R. Kleiss et al., Part. Accel., 41:117-132, 1993.

[5] D. T. Abell. PhD thesis, Univ. of Maryland, 1995.

[6] D. T. Abell et al., PRST-AB to be published, 2001.

[7] F. Schmidt. Tech. Rep. CERN SL-AP Beam Phys. Note 53.

[8] E. Forest. private communication.

[9] M. Böge et al., Tech. Rep. LHC Proj. Note 154, 1998. 